\title{
Relação entre os fatores críticos em projetos de desenvolvimento de software e os atributos que determinam o seu sucesso
}

\section{Gédson Mário Borges Dal Forno}

Professor Adjunto do Departamento de Computação Aplicada da Universidade Federal de Santa Maria (UFSM)

Doutorando em Administração pela Universidade Federal de Santa Maria (UFSM) Rio Grande do Sul, Brasil gedsondf@gmail.com

Felipe Martins Müller

Doutor em Engenharia Elétrica pela Universidade Estadual de Campinas (UNICAMP)

Professor Titular do Departamento de Computação Aplicada da Universidade Federal de Santa Maria (UFSM)

Atua junto ao Programa de Pós-Graduação em Administração da UFSM

Rio Grande do Sul, Brasil

felipe@inf.ufsm.br

Editor Científico: José Edson Lara

Organização Comitê Científico

Double Blind Review pelo SEER/OJS

Recebido em 20.06.2017

Aprovado em 19.07.2017

Este trabalho foi licenciado com uma Licença Creative Commons - Atribuição - Não Comercial 3.0 Brasil 


\title{
Resumo
}

Automatizar processos, por meio do uso de software, tornou-se uma prática amplamente utilizada nos mais diversos níveis organizacionais. Nesse contexto, o sucesso de projetos de desenvolvimento de software passou a ter um papel fundamental e determinante. A importância da definição dos atributos de sucesso, bem como da determinação e tratamento dos fatores críticos em projetos de desenvolvimento de software, pode ser evidenciada através dos diversos estudos já realizados. Este trabalho teve como objetivo verificar as possíveis relações existentes entre os fatores críticos em projetos de desenvolvimento de software e os atributos que determinam seu sucesso. O emprego de métodos estatísticos aos resultados obtidos de uma survey research, aplicada aos profissionais de TI de organizações brasileiras, possibilitou verificar a existência de relações entre os fatores críticos, entre os atributos de sucesso e entre os fatores críticos e os atributos de sucesso. As relações entre os atributos de sucesso justificam, para essas organizações, sua utilização como indicadores do sucesso para projetos de desenvolvimento de software.

Palavras-chave: Fatores Críticos; Sucesso; Fracasso; Projetos de Desenvolvimento de Software; Atributos de Sucesso.

\section{Relationship between critical factors in software development projects and the attributes that determine their success.}

\begin{abstract}
Automating processes, through the use of software, has become a widely used practice at the most diverse organizational levels. In this context, the success of software development projects has played a fundamental and determinant role. The importance of the definition of success attributes, as well as the determination and treatment of critical factors in software development projects, can be evidenced through several studies already carried out. This work aimed to verify the possible relationships between critical factors in software development projects and the attributes that determine their success. The use of statistical methods, to the results obtained from a survey research, applied to the IT professionals of Brazilian organizations, allowed to verify the existence of relations between the critical factors, between the success attributes and between the critical factors and the attributes of success. The relationships between success attributes justify, for these organizations, their use as indicators of success for software development projects.
\end{abstract}

Keywords: Critical Factors; Success; Failure; Software Development Projects; Success Attributes. 


\section{Relación entre los factores críticos y los atributos que determinan el éxito de los proyectos de desarrollo de software.}

\section{Resumen}

La automatización de procedimientos en los más diversos niveles organizacionales valiéndose de software se han convertido en una práctica ampliamente utilizada. En este contexto, el propio éxito de los proyectos de desarrollo de los softwares utilizados pasa a tener papel fundamental y determinante. La importancia de la definición de los denominados Atributos de Éxito (AE), así como de la determinación y tratamiento de los Factores Críticos (FC) en proyectos de desarrollo de software puede ser evidenciada a través de los diversos estudios ya realizados. En ese sentido, este trabajo tuvo como objetivo, por médio del tratamiento estadístico de una encuesta aplicada a profesionales de TI de organizaciones brasileñas, verificar la relación existente entre los propios $\mathrm{FC}$, así como entre los $\mathrm{AE}$ y entre ellos (FC y $\mathrm{AE}$ ) en proyectos de desarrollo de software. Las relaciones entre los atributos de éxito justifican, para estas organizaciones, su utilización como indicadores del éxito para proyectos de desarrollo de software.

Palabras clave: Factores Críticos; Éxito; Fracaso; Proyectos de Desarrollo de Software; Atributos de Éxito.

\section{Introdução}

Atualmente, os Sistemas de Informações (SI) automatizados vêm sendo amplamente utilizados nos mais diversos setores das organizações. Isso se justifica pelo fato de a automação de processos, realizada através da utilização de software, torná-los rápidos e eficientes, constituindo-se em ferramentas essenciais no auxílio à tomada de decisão nos mais diversos níveis organizacionais, de forma a atender as constantes mudanças responsáveis pelo ambiente dinâmico a que estão sujeitas.

Tendo em vista a importância das informações resultantes dos SI, torna-se necessário que esses apresentem disponibilidade e confiabilidade, em que disponibilidade é a probabilidade de um SI estar operando com sucesso, de acordo com as especificações, em um determinado momento, e confiabilidade é a probabilidade de um SI operar sem apresentar defeitos, sob certas condições, em um determinado intervalo de tempo (Pfleeger, 2004, p. 330).

Sendo o software um dos componentes das Tecnologias da Informação (Laudon \& Laudon, 2010) e responsável pela automação dos processos, também deve possuir essas características. Um software que apresente disponibilidade e 
confiabilidade pode ser obtido através da aplicação dos métodos existentes para o desenvolvimento de projetos de software, o que implica resultados de sucesso para esses projetos.

Porém, embora o software continue sendo desenvolvido através de projetos que utilizam algum desses métodos, ou até mesmo por um método criado pela própria organização, muitos deles ainda continuam a apresentar resultados de falhas.

Para o Standish Group (2013), os projetos de software, em relação à sua resolução, são classificados em três tipos: Projetos de Sucesso, Projetos Contestados e Projetos Fracassados.

De acordo com o Standish Group (2013), embora tenha ocorrido um aumento considerável na taxa de resolução de projetos de sucesso, as taxas relativas aos projetos contestados e fracassados permaneceram preocupantes, merecendo uma continuidade de investigações.

Com o objetivo de melhorar essas taxas e buscando sempre direcionar os resultados desses projetos para o sucesso, muitos estudos vêm sendo desenvolvidos abordando Fatores Críticos de Sucesso e Fatores Críticos de Falhas em projetos de desenvolvimento de software. Entretanto, parte desses estudos não apresenta de forma clara qual a definição utilizada para sucesso e/ou fracasso. Outros trabalhos atribuem à importância dos fatores estudados apenas baseados na sua frequência de citação na literatura. Em alguns desses estudos, os fatores encontrados são agrupados aleatoriamente em categorias, para as quais também não existe um consenso quanto à nomenclatura.

Com base nessas premissas, após determinar os Fatores Críticos que atuam nos resultados de projetos de desenvolvimento de software, assim como os Atributos utilizados para definir o sucesso do resultado desses projetos (Müller \& Dal Forno, 2016), objetivou-se, neste trabalho, verificar a relação existente entre estes Fatores Críticos e estes Atributos, utilizados para definir o sucesso, em projetos de desenvolvimento de software executados em organizações brasileiras.

\section{Referencial Teórico}

Vários estudos têm sido realizados sobre os fatores críticos de sucesso e de fracasso em projetos, entretanto, de acordo com Sudhakar (2012), os projetos de 
software apresentam diferenças em relação aos projetos tradicionais, uma vez que apresentam características que os tornam mais críticos e complexos.

O desenvolvimento de projetos de software envolve fatores cruciais, como: confiabilidade, sigilo, prestação de contas, disponibilidade de documentação e integridade, além de interesses e prioridades próprias, das várias partes envolvidas, poderem impactar o sucesso do projeto. Dessa maneira, a combinação dessas características pode variar de projeto para projeto, sugerindo que a importância dos diferentes fatores críticos de sucesso também será afetada (Ahimbisibwe, Cavana, \& Daellenbach, 2015).

Nasir e Sahibuddin (2011) salientam a influência do processo nos resultados de projetos de desenvolvimento de software, o que é referendado por Ahimbisibwe et al. (2015) ao argumentar que pesquisas anteriores em gerenciamento de projetos têm contribuído para identificar os fatores críticos que influenciam o resultado (sucesso ou fracasso) de projetos de software; mesmo assim, não existe um amplo consenso sobre esses fatores críticos. Ainda segundo por Ahimbisibwe et al. (2015), os esforços dessas pesquisas têm sido relacionados aos resultados de projetos de desenvolvimento de software, e não ao processo de desenvolvimento de software em si.

Para embasar estudo anterior, no qual definiram os Fatores Críticos para o resultado de projetos de desenvolvimento de software, Müller e Dal Forno (2016) utilizaram estudos e relatórios cujo foco eram fatores críticos de sucesso e fatores críticos de falha. A Tabela 1 apresenta a frequência das variáveis encontradas na revisão bibliográfica, bem como a identificação dos estudos e/ou relatórios em que foram empregadas. 
Tabela 1

Fatores críticos para o resultado de projetos de desenvolvimento de software

\begin{tabular}{|c|c|c|c|}
\hline \multirow{2}{*}{ № } & \multirow{2}{*}{ Fatores Críticos } & \multirow{2}{*}{ Freq. } & Referências - Sucesso \\
\hline & & & Referências - Falhas \\
\hline \multirow{2}{*}{1} & \multirow{2}{*}{ Gestão do projeto } & \multirow{2}{*}{14} & {$[02],[04],[05],[07],[08],[10],[12],[15]$} \\
\hline & & & {$[01],[03],[02],[04],[06],[11]$} \\
\hline \multirow{2}{*}{2} & \multirow{2}{*}{ Especificação de requisitos } & \multirow{2}{*}{13} & {$[02],[04],[05],[09],[11],[15]$} \\
\hline & & & {$[01],[02],[03],[04],[06],[11],[13]$} \\
\hline \multirow[b]{2}{*}{3} & \multirow{2}{*}{ Apoio da gestão superior } & \multirow{2}{*}{13} & {$[02],[04],[05],[07],[08],[10],[11],[15]$} \\
\hline & & & {$[01],[04],[06],[11],[13]$} \\
\hline \multirow{2}{*}{4} & \multirow{2}{*}{ Definição de objetivos } & \multirow{2}{*}{13} & {$[02],[04],[05],[07],[08],[09],[10],[11]$} \\
\hline & & & {$[02],[03],[06],[11],[13]$} \\
\hline \multirow{2}{*}{5} & \multirow{2}{*}{$\begin{array}{l}\text { Capacidade e competência da } \\
\text { equipe }\end{array}$} & \multirow{2}{*}{11} & {$[02],[04],[05],[07],[08],[11],[12],[15]$} \\
\hline & & & {$[01],[04],[06]$} \\
\hline \multirow{2}{*}{6} & \multirow{2}{*}{ Comunicação no projeto } & \multirow{2}{*}{11} & [02], [04], [05], [07], [09], [12], [15] \\
\hline & & & {$[01],[04],[06],[13]$} \\
\hline \multirow{2}{*}{7} & \multirow{2}{*}{ Planejamento do projeto } & \multirow{2}{*}{11} & {$[04],[05],[07],[09],[10],[11],[15]$} \\
\hline & & & {$[02],[03],[04],[11]$} \\
\hline \multirow{2}{*}{8} & \multirow{2}{*}{ Apoio e recursos } & 10 & {$[05],[07],[10],[12],[15]$} \\
\hline & & 10 & {$[01],[04],[06],[11],[13]$} \\
\hline 0 & Fnyolvimonto do ucuório & 10 & {$[04],[05],[07],[08],[10],[11]$} \\
\hline 9 & Envolvimento do usuario & 10 & {$[01],[03],[06],[11]$} \\
\hline 10 & Gestãn de mudancas & 9 & [05], [09], [15] \\
\hline 10 & Gestao de munariças & & [01], [03], [04], [06], [11], [13] \\
\hline 11 & Cronograma do nroieto & 9 & {$[02],[05],[07],[09],[15]$} \\
\hline 11 & Cronograma do projeto & 9 & {$[01],[03],[11],[13]$} \\
\hline 10 & Infraestrutura $\mathrm{e}$ ferramentas $\mathrm{de}$ & 0 & [02], [04], [05], [08], [15] \\
\hline 12 & apoio & 8 & {$[02],[04],[06]$} \\
\hline 13 & Comprometimento e motivação da & 7 & {$[02],[04],[05],[11],[12],[15]$} \\
\hline & equipe do projeto & & [01] \\
\hline 14 & Tamanho do nroieto & 7 & {$[04],[05],[08],[11],[15]$} \\
\hline 14 & & 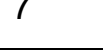 & {$[03],[06]$} \\
\hline 15 & Particinacão do usuárin & 7 & {$[02],[15]$} \\
\hline & & $f$ & [01], [04], [06], [11], [13] \\
\hline & & 7 & {$[04],[07],[11]$} \\
\hline 16 & Expectatıvas do usuarıo & 7 & {$[03],[04],[06],[11]$} \\
\hline 17 & Metodologia de decenvolvimento & 6 & {$[05],[08],[12],[15]$} \\
\hline & & 6 & {$[03],[06]$} \\
\hline 18 & Incertezas tecnológicas & 5 & {$[07],[15]$} \\
\hline & Incertezas tecnologıcas & 5 & [04], [06], [11] \\
\hline 19 & Cultura orranizacional & 5 & [02], [07], [15] \\
\hline 19 & Cuitura organizacional & 5 & {$[02],[06]$} \\
\hline 20 & Composicão da equipe do projeto & 5 & [02], [07], [15] \\
\hline & & 5 & {$[03],[06]$} \\
\hline
\end{tabular}


(conclusão)

\begin{tabular}{|c|c|c|c|}
\hline № & Fatores Críticos & Freq. & $\begin{array}{l}\text { Referências - Sucesso } \\
\text { Referências - Falhas }\end{array}$ \\
\hline \multirow{2}{*}{21} & \multirow{2}{*}{ Monitoramento e controle do projeto } & \multirow{2}{*}{5} & [05], [07], [12], [14] \\
\hline & & & [02] \\
\hline \multirow{2}{*}{22} & \multirow{2}{*}{ Relacionamento equipe/usuário } & \multirow{2}{*}{4} & [02], [07], [08] \\
\hline & & & [02] \\
\hline \multirow{2}{*}{23} & \multirow{2}{*}{ Complexidade do projeto } & \multirow{2}{*}{4} & [05], [08], [15], \\
\hline & & & [06] \\
\hline \multirow{2}{*}{24} & \multirow{2}{*}{ Orçamento do projeto } & \multirow{2}{*}{4} & [05], [09], [15] \\
\hline & & & [03] \\
\hline 25 & Liderança do projeto & 3 & [05], [07], [15] \\
\hline \multirow{2}{*}{26} & \multirow{2}{*}{ Análise e gestão de riscos } & \multirow[b]{2}{*}{3} & [05], [15] \\
\hline & & & {$[03]$} \\
\hline \multirow{2}{*}{27} & \multirow{2}{*}{ Suporte ao usuário } & \multirow{2}{*}{3} & [05], [15] \\
\hline & & & [06] \\
\hline \multirow{2}{*}{28} & \multirow{2}{*}{$\begin{array}{l}\text { Comprometimento da gestão } \\
\text { superior }\end{array}$} & \multirow{2}{*}{3} & [12] \\
\hline & & & [03], [06] \\
\hline 29 & Criticidade do projeto & 2 & [02], [15] \\
\hline \multirow{2}{*}{30} & \multirow{2}{*}{ Controle de qualidade } & \multirow{2}{*}{2} & [02], [15] \\
\hline & & & \\
\hline 31 & Processo de testes & 1 & [02] \\
\hline 32 & Tamanho da equipe do projeto & 1 & \\
\hline
\end{tabular}

\section{LEGENDA}

\begin{tabular}{l|l}
\hline [01] Kappelman, McKeeman e Zhang (2006) & [02] Chow e Cao (2008)
\end{tabular}

[03] Verner, Sampson e Cerpa (2008) $\quad$ [04] Rukshan e Mangala (2010)

[05] Nasir e Sahibuddin (2011) $\quad$ [06] McLeod e MacConell (2011)

[07] Sudhakar (2012) $\quad$ [08] Standish Group (2013)

[09] Hashim, Abbas e Hashim (2013) [10] Marques, Varjão, Sousa e Peres (2013)

[11] Standish Group (2014) $\quad$ [12] Kouzari, Gerogiannis, Stamelos e

\begin{tabular}{ll} 
& Kakarontzas (2015) \\
\hline [13] Sweis (2015) & [14] Ahimbisibwe et al. (2015)
\end{tabular}

Nota. Fonte: Elaborada pelos autores.

Ao observarem que muitos fatores citados na literatura como fatores críticos de sucesso também eram citados na literatura como fatores críticos de fracasso, os autores propuseram a não divisão em fatores críticos de sucesso e em fatores críticos de falhas, mas apenas a existência de Fatores Críticos, os quais, de acordo com a atenção e o tratamento que Ihes forem dispensados, poderão levar os resultados de projetos de desenvolvimento de software a ser um sucesso, contestados ou fracassados (entendimento esse sintetizado na Figura 1).

A bibliografia utilizada leva a algumas considerações importantes a respeito do estudo de Fatores Críticos para o resultado de projetos de desenvolvimento de software. Em primeiro lugar, pode ser citada a inexistência de uma definição clara e padrão para sucesso e fracasso no âmbito considerado pelo estudo, conforme pode ser visto em McLeod e MacDonell (2011), Sudhakar (2012), Kaur e Aggrawal (2013) 
e Ahimbisibwe et al. (2015). Em razão da variedade de conceitos e definições existentes, torna-se necessário que os autores deixem claro, em seus estudos, qual a definição ou conceito adotado.

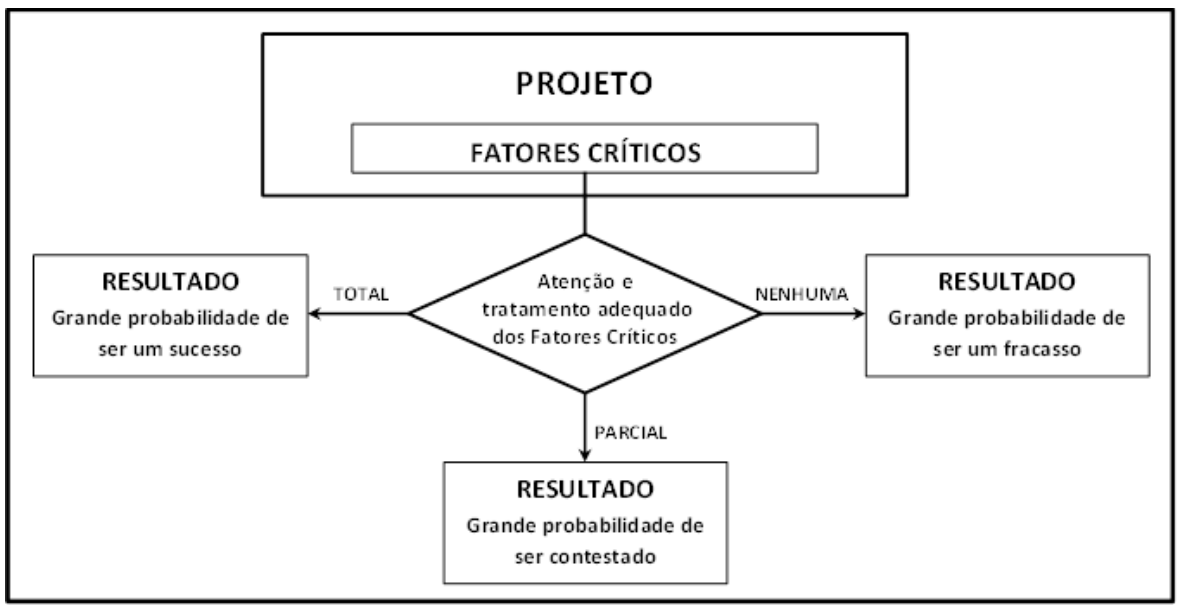

\section{Figura 1}

Probabilidades do resultado de projetos de desenvolvimento de software Fonte: elaborada pelos autores.

Neste estudo, optou-se por utilizar os atributos de sucesso, conforme apresentado na Tabela 2, os quais representam exatamente a percepção geral de sucesso do resultado de um projeto em particular. Atributos esses utilizados por Chow e Cao (2008) e também referenciados, total ou parcialmente, em estudos como Rukshan e Mangala (2010), Hashin, Abbas e Hashin (2013), Marques et al. (2013), Standish Group (2013), Standish Group (2014) e Sweis (2015).

\section{Tabela 2}

Atributos de sucesso

\begin{tabular}{|c|c|}
\hline DIMENSĀO & ATRIBUTO \\
\hline \multirow{4}{*}{$\begin{array}{l}\text { Nível geral de percepção } \\
\text { de sucesso }\end{array}$} & $\begin{array}{l}\text { Qualidade (entregar um bom produto }- \text { correto e } \\
\text { operacional) }\end{array}$ \\
\hline & $\begin{array}{l}\text { Escopo (cumprir com todos os requisitos e objetivos } \\
\text { estabelecidos) }\end{array}$ \\
\hline & Tempo (entregar dentro do cronograma estabelecido) \\
\hline & Custo (entregar dentro do orçamento e do esforço estimado) \\
\hline
\end{tabular}

Nota. Fonte: Recuperado de "A Survey Study of Critical Factors in Agile Software Projects," Chow, T. \& Cao, D. B., 2008, Journal of System and Software, 81(6), p. 963.

Ahimbisibwe et al. (2015) salientam que o fato de os resultados de estudos serem específicos de um determinado país, além da diferença de tamanho, de domínio e da complexidade dos projetos considerados nos estudos, pode ser a causa da falta de um amplo consenso a respeito dos fatores críticos de sucesso e de falhas em projetos de desenvolvimento de software. 
Isso também é enfatizado por Nasir e Sahibuddin (2011), quando alegam a necessidade do desenvolvimento de estudos envolvendo diferentes tamanhos de projetos em vários domínios e países.

Em razão disso, este estudo foi desenvolvido tendo como público-alvo os profissionais de organizações brasileiras envolvidos em projetos de desenvolvimento de software, sendo utilizados os seguintes Fatores Críticos: Características da Equipe, Planejamento do Projeto, Metodologia de Desenvolvimento, Esforços Organizacionais, Atitudes do Usuário/Cliente, Administração de Recursos, Características do Projeto e Qualidade do Software, todos esses definidos por Müller e Dal Forno (2016) em seu estudo, que também envolveu o mesmo tipo de profissionais e organizações.

\section{Metodologia}

O percurso metodológico utilizado neste trabalho, conforme pode ser visualizado na Figura 2, constou dos seguintes passos: revisão da literatura, realização de uma survey research (pesquisa de levantamento), determinação dos Fatores Críticos e dos Atributos de Sucesso e, finalmente, a verificação das relações entre eles.

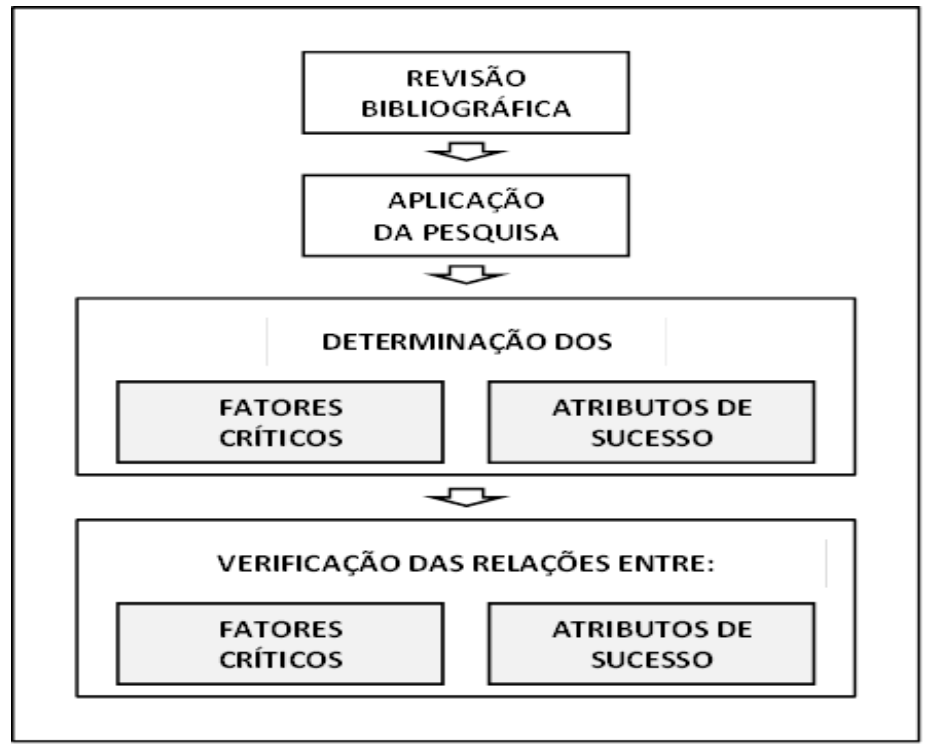

Figura 2

Percurso metodológico

Fonte: Os autores. 
Para a revisão da literatura, inicialmente realizou-se um levantamento bibliográfico cuja finalidade era verificar e selecionar a literatura existente sobre 0 assunto. Tendo em vista que este trabalho alicerçou-se no estudo de Nasir e Sahibuddin (2011), optou-se por utilizar as mesmas bases de dados online de revistas que foram utilizadas nesse estudo, ou seja: www.sciencedirect.com, www.ieeexplore.ieee.org, www.springerlink.com e www.emeraldinsight.com.

Os critérios de busca utilizados foram: artigos publicados a partir de 2011, uma vez que o período da pesquisa realizada por Nasir e Sahibuddin (2011) foi de 1990 a 2010; artigos que continham no título "development projects" e "information systems" e/ou "IS" e/ou "software" e/ou "information technology" e/ou "IT"; além disso, os artigos deveriam conter as palavras-chave: "critical factors of failure" e/ou "failure factors" e/ou "critical success factors" e/ou "success factors".

A pesquisa ocorreu no período compreendido entre abril de 2015 e agosto de 2016, retornando 21 artigos, sendo que 12 foram retirados por não tratarem especificamente do assunto de interesse, restando, portanto, 9 artigos. Também foram incluídos 3 artigos e 2 relatórios do Standish Group citados em suas referências, sendo esses considerados importantes para o assunto objeto deste estudo.

Posteriormente, realizou-se uma survey research (pesquisa de levantamento), na qual foi aplicado o instrumento desenvolvido e validado em Müller e Dal Forno (2016), sendo esse disponibilizado online, por um período de 90 dias, para aproximadamente 900 profissionais da área de desenvolvimento de projetos de software. Esses profissionais foram convidados a participar da pesquisa por meio de e-mail, cujos endereços foram localizados na Internet ou através de associações e sindicatos de empresas e de profissionais da área. Obteve-se um retorno de 172 questionários, valor correspondente a, aproximadamente, 19\% dos e-mails enviados.

Através do feedback dos participantes, foi possível a Müller e Dal Forno (2016) verificarem a percepção deles (participantes) em relação aos Atributos de Sucesso (Figura 3), adotados por Chow e Cao (2008) e utilizados neste estudo, bem como determinar os Fatores Críticos (Tabela 3) que contribuem para o resultado de projetos de desenvolvimento de software.

No que diz respeito à média dos Atributos de Sucesso, conforme pode ser observado na Figura 3 e, tendo em vista que o instrumento aplicado utilizava uma 
escala Likert de 5 pontos ( 1 - muito baixa a 5 - muito alta), percebe-se que os quatro atributos obtiveram médias elevadas, indicando que, de acordo com a percepção dos entrevistados, esses atributos apresentam um alto grau de importância.

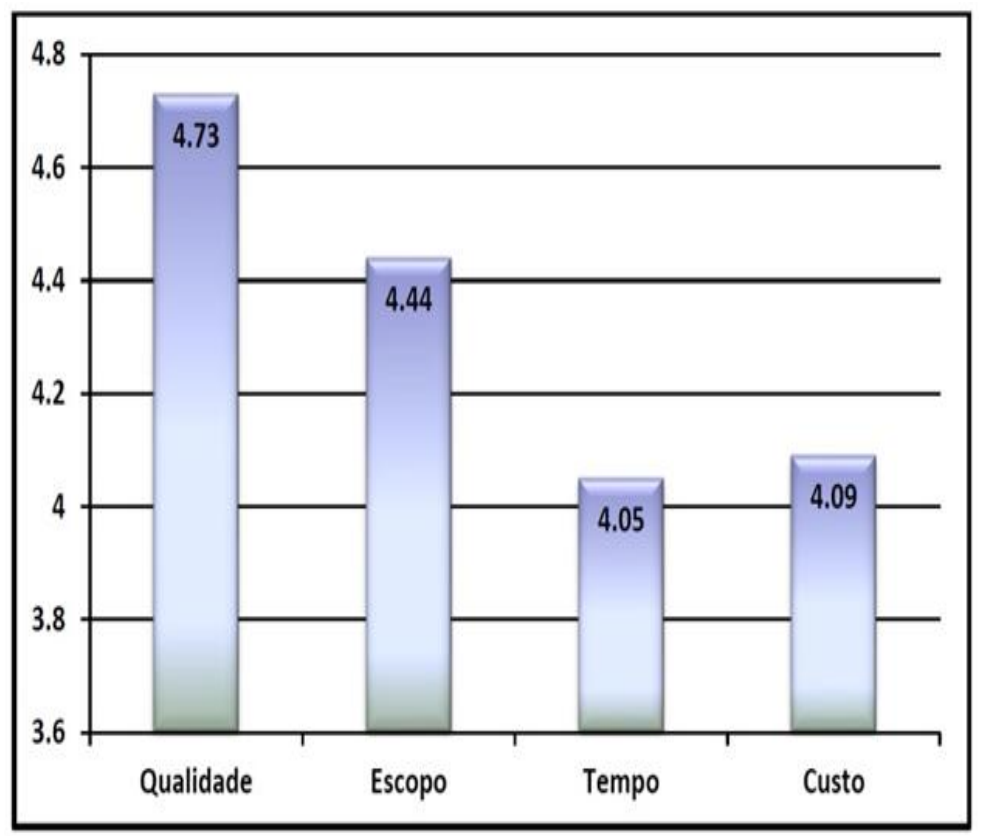

\section{Figura 3}

Médias dos atributos de sucesso

Fonte: Dados da pesquisa

\section{Tabela 3}

Fatores críticos

\begin{tabular}{|c|c|}
\hline Fatores & Variáveis \\
\hline \multirow{5}{*}{$\begin{array}{l}\text { Características da } \\
\text { Equipe }\end{array}$} & Capacidade e Competência da Equipe \\
\hline & Experiência da Equipe \\
\hline & Comprometimento e Motivação da Equipe \\
\hline & Experiência e Competência do Gestor do Projeto \\
\hline & Relacionamento Equipe/Usuário \\
\hline \multirow{6}{*}{$\begin{array}{l}\text { Planejamento do } \\
\text { Projeto }\end{array}$} & Especificação de Requisitos \\
\hline & Definição de Objetivos e Metas \\
\hline & Planejamento do Cronograma \\
\hline & Planejamento do Projeto \\
\hline & Gestão de Riscos (Análise Prévia e Tratamento) \\
\hline & Treinamento e Aprendizagem de Usuários/Clientes \\
\hline \multirow{6}{*}{$\begin{array}{l}\text { Metodologia de } \\
\text { Desenvolvimento }\end{array}$} & Metodologia de Desenvolvimento \\
\hline & Tamanho da Equipe \\
\hline & Composição da Equipe \\
\hline & Experiência da Equipe com a Metodologia Utilizada \\
\hline & Processo e Metodologia Adequados \\
\hline & Infraestrutura e Ferramentas de Apoio \\
\hline \multirow{5}{*}{$\begin{array}{l}\text { Esforços } \\
\text { Organizacionais }\end{array}$} & Apoio da Gestão Superior \\
\hline & Comprometimento da Gestão Superior \\
\hline & Liderança do Projeto \\
\hline & Recursos Adequados (Humanos, Financeiros e Materiais) \\
\hline & Organizacional (do Cliente) \\
\hline
\end{tabular}




\begin{tabular}{|c|c|}
\hline \multirow{2}{*}{$\begin{array}{ll}\text { Atitudes } & \text { do } \\
\text { Usuário/Cliente }\end{array}$} & Participação do Usuário/Cliente \\
\hline & Comprometimento do Usuário/Cliente \\
\hline \multirow{4}{*}{$\begin{array}{l}\text { Administração } \\
\text { Recursos }\end{array}$} & Expectativas Realistas do Usuário/Cliente \\
\hline & Comunicação Interna do Projeto \\
\hline & Estimativas de Custos Previamente Realizadas \\
\hline & Orçamento Realista \\
\hline \multirow{2}{*}{$\begin{array}{l}\text { Características } \mathrm{d} \\
\text { Projeto }\end{array}$} & Complexidade do Projeto \\
\hline & Tamanho do Projeto \\
\hline \multirow{3}{*}{$\begin{array}{l}\text { Qualidade } \\
\text { Software }\end{array}$} & Monitoramento e Controle Efetivo do Projeto \\
\hline & Controle de Qualidade \\
\hline & Processo de Testes \\
\hline
\end{tabular}

Nota. Fonte: Elaborada pelos autores.

Já em relação às variáveis utilizadas nesta pesquisa para determinar os fatores críticos em projetos de desenvolvimento de software, salienta-se que estão presentes na maioria dos estudos utilizados como referências, entretanto, em muitos deles, aparecem distribuídas em fatores que apresentam nomenclaturas diferentes. Ahimbisibwe et al. (2015) argumentam que, embora considerando as contribuições de pesquisas anteriores, não existe um amplo consenso sobre os fatores críticos e que, de acordo com a perspectiva de risco utilizada, os estudos podem ter identificado fatores críticos semelhantes, mas utilizado nomenclaturas diferentes.

A pesquisa desenvolvida neste estudo teve caráter quantitativo, e o método de pesquisa utilizado foi do tipo survey. Para a tabulação e análise quantitativa dos dados, foi utilizado o software de apoio SPSS 20.0 (Statiscal Package for the Social Sciences), por meio da realização de técnicas de análise estatística descritiva. Com a finalidade de estabelecer a relação entre os Fatores Críticos e os Atributos de Sucesso, utilizou-se do Coeficiente de Correlação de Pearson, o qual, de acordo com Hair, Black, Babin, Anderson e Tatham (2009), indica a força de associação entre quaisquer duas variáveis.

Lopes (2016) explica que o coeficiente de Pearson assume valores negativos e positivos que variam de $-1 \mathrm{a}+1$, passando por zero. Logo, valores próximos de -1 significam pouca dispersão e uma correlação forte e negativa, enquanto próximos de "zero" indicam muita dispersão e ausência de relação entre as variáveis e, ainda, quando próximos de +1 , significam pouca dispersão e uma correlação forte e positiva.

Com relação à intensidade das correlações, por convenção, Pestana e Gageiro (2008) sugerem que as variações do coeficiente de Correlação de Pearson entre 0,01 e 0,2 são ditas muito baixas; entre 0,2 e 0,39, são classificadas como 
baixas; entre 0,4 e 0,69, são consideradas moderadas; entre 0,7 e 0,89, são designadas altas; e entre 0,9 e 1, são qualificadas como muito altas.

Após a conclusão da tabulação e da análise quantitativa dos dados, realizaram-se, na seção 4 , a descrição e a análise dos resultados obtidos.

\section{Apresentação e Discussão dos Resultados}

Os resultados encontrados neste trabalho estão descritos e analisados na subseção 4.1, que aborda o perfil dos respondentes, e na subseção 4.2, que trata a relação entre os Fatores Críticos e os Atributos de Sucesso em projetos de desenvolvimento de software.

\subsection{Perfil dos respondentes}

A amostra utilizada no presente estudo foi composta por 172 respondentes, profissionais da área de desenvolvimento de projetos de software atuando no Distrito Federal e em 18 estados do Brasil.

Com relação ao perfil dos respondentes, a faixa etária concentrou-se entre 26 e 35 anos, perfazendo um total de $47 \%$ da amostra; a escolaridade apresentou uma maior concentração na pós-graduação completa, com um total de $41 \%$ da amostra; e o nível de direção, com $50 \%$ da amostra, apresentou a maior concentração para o cargo ocupado na empresa.

No que diz respeito ao perfil dos projetos, tomados como referência pelos entrevistados para responder às questões de pesquisa, o resultado do projeto apresentou praticamente um equilíbrio, concentrando-se no sucesso com $55 \%$ e no contestado com $44 \%$ da amostra. Similarmente, o ano de conclusão do projeto apresentou maior concentração em 2016 , com $50 \%$, e em 2015 , com $40 \%$ do total da amostra. Já a função desempenhada no projeto apresentou uma concentração de $59 \%$ da amostra tanto para gerentes do projeto como para desenvolvedores (analista, projetista, programador, engenheiro de testes), salientando-se aqui a possibilidade de o respondente assumir mais de uma função no projeto.

\subsection{Relação entre os Fatores Críticos e os Atributos de Sucesso em Projetos de Desenvolvimento de Software}


Com o propósito de estabelecer as relações entre os Fatores Críticos e os Atributos de Sucesso (ver convenção utilizada para identificação na Tabela 4) em projetos de software, de acordo com as percepções dos profissionais de $\mathrm{TI}$ entrevistados, recorreu-se à análise de correlação entre os construtos. Dessa forma, calculou-se o coeficiente de Correlação de Pearson.

Por meio da análise dos dados resultantes do cálculo do coeficiente de Correlação de Pearson, apresentados na Tabela 5, é possível observar que, de uma maneira geral, foram obtidos coeficientes de correlação significativos, os quais, por serem todos positivos, indicam que as variáveis possuem o mesmo sentido de associação. Além disso, é possível notar que, em $82 \%$ dos casos significativos, obtiveram-se coeficientes de correlação entre 0,2 e 0,69, indicando que grande parte das correlações possui intensidade baixa a moderada (Pestana \& Gageiro, 2008).

\section{Tabela 4}

Identificação dos fatores críticos e dos atributos de sucesso

\begin{tabular}{|c|c|}
\hline \multicolumn{2}{|c|}{ FATORES CRITICOS } \\
\hline Denominação & Descrição \\
\hline Fat1 & Características da Equipe \\
\hline Fat2 & Planejamento do Projeto \\
\hline Fat3 & Metodologia de Desenvolvimento \\
\hline Fat4 & Esforços Organizacionais \\
\hline Fat5 & Atitudes do Usuário/Cliente \\
\hline Fat6 & Administração de Recursos \\
\hline Fat7 & Características do Projeto \\
\hline Fat8 & Qualidade do Software \\
\hline \multicolumn{2}{|c|}{ ATRIBUTOS DE SUCESSO } \\
\hline Denominação & Descrição \\
\hline Atr1 & Qualidade: entregar um bom produto - correto e operacional \\
\hline Atr2 & $\begin{array}{l}\text { Escopo: cumprir com todos os requisitos e objetivos } \\
\text { estabelecidos }\end{array}$ \\
\hline Atr3 & Tempo: entregar dentro do cronograma estabelecido \\
\hline Atr4 & Custo: entregar dentro do orçamento e do esforço estimado \\
\hline
\end{tabular}

Nota. Fonte: Elaborada pelos autores.

Entretanto, destaca-se que não foram significativas as correlações entre as variáveis Fat5 (Atitudes do Usuário/Cliente) e Fat7 (Características do Projeto), Fat1 (Características da Equipe) e Atr2 (Escopo), Fat1 (Características da Equipe) e Atr4 (Custo), Fat3 (Metodologia de Desenvolvimento) e Atr2 (Escopo), Fat3 (Metodologia de Desenvolvimento) e Atr3 (Tempo), Fat3 (Metodologia de Desenvolvimento) e Atr4 (Custo), Fat4 (Esforços Organizacionais) e Atr2 (Escopo), Fat5 (Atitudes do Usuário/Cliente) e Atr1 (Qualidade), Fat5 (Atitudes do Usuário/Cliente) e Atr2 
(Escopo), Fat5 (Atitudes do Usuário/Cliente) e Atr3 (Tempo), Fat6 (Administração de Recursos) e Atr2 (Escopo), Fat6 (Administração de Recursos) e Atr3 (Tempo), Fat7 (Características do Projeto) e Atr2 (Escopo), Fat7 (Características do Projeto) e Atr3 (Tempo), Fat7 (Características do Projeto) e Atr4 (Custo), Fat8 (Qualidade do Software) e Atr2 (Escopo), salientadas com negrito na Tabela 5.

Analisando especificamente a relação entre os fatores que compõem o construto Fatores Críticos, sinalizada na cor cinza claro na Tabela 5, constata-se que apenas a correlação entre o Fat5 (Atitudes do Usuário/Cliente) e o Fat7 (Características do Projeto) foi não significativa. Com relação às intensidades das demais correlações, as quais foram significativas, 40,5\% são consideradas como moderadas, tendo em vista que se encontram no intervalo compreendido entre 0,4 e 0,69, e 59,5\% são consideradas baixas, pois se encontram no intervalo de 0,2 a 0,39 (Pestana \& Gageiro, 2008).

\section{Tabela 5}

Matriz de correlação entre os fatores críticos e os atributos de sucesso

\begin{tabular}{|c|c|c|c|c|c|c|c|c|c|c|c|c|}
\hline Correl. & Fat1 & Fat2 & Fat3 & Fat4 & Fat5 & Fat6 & Fat7 & Fat8 & Atr1 & Atr2 & Atr3 & Atr4 \\
\hline Fat1 & 1 & 0,347 & 0,675 & 0,469 & 0,376 & 0,481 & 0,211 & 0,448 & 0,189 & 0,028 & 0,162 & 0,137 \\
\hline Fat2 & & 1 & :* 0,344 & ${ }^{* *} 0,483$ & $\begin{array}{l}\star * \\
0,301\end{array}$ & 0,489 & $\begin{array}{l}* * \\
0,369\end{array}$ & ${ }^{* \star}$ & $\begin{array}{l}\star * \\
0,263\end{array}$ & *** 0,290 & 0,244 & $\begin{array}{l}{ }^{* \star} \\
0,222\end{array}$ \\
\hline Fat3 & & & 1 & $\begin{array}{l}* * \\
0,524\end{array}$ & $\begin{array}{l}* * \\
0,242\end{array}$ & $\begin{array}{l}\star * \\
0,392\end{array}$ & $\begin{array}{l}* * \\
0,226\end{array}$ & $\begin{array}{l}* * \\
0,491\end{array}$ & $\begin{array}{l}* \\
0,186\end{array}$ & $\overline{0,006}$ & $\bar{x}_{\star \star}^{0,112}$ & $\overline{0,133}$ \\
\hline Fat4 & & & & 1 & 0,373 & 0,342 & 0,245 & 0,463 & 0,179 & 0,025 & 0,201 & 0,206 \\
\hline Fat5 & & & & & 1 & $\begin{array}{l}\star \star * \\
0,398\end{array}$ & $\overline{0,134}$ & $\begin{array}{l}* * \\
0,279\end{array}$ & $\overline{0,127}$ & $\overline{0,068}$ & $\overline{0,087}$ & $\begin{array}{l}* \\
0,152\end{array}$ \\
\hline Fat6 & & & & & & 1 & $\begin{array}{l}\star \star \\
0,216\end{array}$ & $\stackrel{* \star}{* \star}, 419$ & $\begin{array}{l}\star * \\
0,211\end{array}$ & $\overline{0,108}$ & $\overline{0,053}$ & 0,164 \\
\hline Fat7 & & & & & & & 1 & $\begin{array}{l}* * \\
0,389\end{array}$ & * 0,163 & $\overline{0,021}$ & $\overline{0,014}$ & $\overline{0,006}$ \\
\hline Fat8 & & & & & & & & 1 & * 0,155 & & & $\begin{array}{l}* * \\
0,259 \\
\end{array}$ \\
\hline Atr1 & & & & & & & & & 1 & $\begin{array}{l}* * \\
0,274\end{array}$ & $\begin{array}{l}* * \\
0,315\end{array}$ & $\begin{array}{l}\text { ** }^{*} \\
0,308\end{array}$ \\
\hline Atr2 & & & & & & & & & & & औ* 0,318 & $\begin{array}{l}* * \\
0,197\end{array}$ \\
\hline Atr3 & & & & & & & & & & & 1 & $\begin{array}{l}* * \\
0,618\end{array}$ \\
\hline Atr4 & & & & & & & & & & & & 1 \\
\hline
\end{tabular}

Ressalta-se, ainda, que o maior coeficiente de correlação obtido $(0,675)$ se refere à associação entre o Fat1 (Características da Equipe) e o Fat3 (Metodologia de Desenvolvimento). Esse resultado demonstra a importância do conhecimento, da 
competência e da experiência dos membros da equipe, assim como do gestor do projeto em relação à metodologia escolhida para o desenvolvimento do projeto.

No que diz respeito à correlação dos Atributos de Sucesso, marcada na cor cinza médio na Tabela 5, verifica-se que todos os coeficientes de correlação foram significativos, o que justifica a utilização desses atributos como indicadores do sucesso. De modo geral, levando em considerando as intensidades, a maioria dos coeficientes encontrados sinaliza uma associação baixa entre as dimensões, com exceção da correlação entre o Atr3 (Tempo) e o Atr4 (Custo), que apresentou um coeficiente de correlação moderado $(0,618)$, o que indica a importância de um cronograma bem estabelecido para a observância do orçamento e dos recursos estimados.

Ao considerar o relacionamento entre a percepção dos Fatores Críticos e das dimensões dos Atributos de Sucesso, destacado na cor cinza escuro na Tabela 5, pode-se afirmar que os resultados demonstram, em todos os casos, a existência de correlações baixas. Contudo, cabe evidenciar que as dimensões Fat2 (Planejamento do Projeto) e Atr2 (Escopo) exibiram o maior coeficiente de correlação entre os construtos $(0,290)$, o que remete ao entendimento de que quanto maior a atenção dispensada ao planejamento do projeto (especificação de requisitos, definição de objetivos e metas, planejamento de cronograma, gestão de riscos, treinamento e aprendizado de usuários/clientes), maior será a possibilidade de atender o atributo de sucesso Escopo (cumprir todos os requisitos e objetivos estabelecidos).

\section{Conclusões}

Em estudo anterior, Müller e Dal Forno (2016), além de determinarem os Fatores Críticos para o resultado de projetos de desenvolvimento de software em organizações brasileiras, verificaram qual a percepção dos profissionais dessas organizações em relação ao conceito de sucesso (Atributos do Sucesso) adotado por Chow e Cao (2008).

Dando continuidade ao estudo de Müller e Dal Forno (2016), com a finalidade de torná-lo mais completo, objetivou-se, neste trabalho, verificar, para as organizações brasileiras de desenvolvimento de software, a existência de relações entre os Fatores Críticos para o resultado de projetos de desenvolvimento de software e os atributos que determinam seu sucesso. O processo de verificação 
dessas relações constou das seguintes etapas: a) determinação do coeficiente de correlação de Pearson; b) verificação das relações entre os Fatores Críticos, entre os Atributos do Sucesso e entre os Fatores Críticos e os Atributos do Sucesso; e c) de uma análise dessas relações.

Encerrada a execução das etapas acima descritas, foi possível verificar, para as organizações brasileiras de desenvolvimento de software, a existência de relações entre os Fatores Críticos, entre os Atributos do Sucesso e entre os Fatores Críticos e os Atributos do Sucesso.

Além disso, por meio das relações existentes entre os Atributos de Sucesso (Qualidade, Escopo, Tempo e Custo), foi possível justificar, para essas organizações, sua utilização como identificadores do sucesso de resultados de projetos de desenvolvimento de software.

Paralelamente, este trabalho também permitiu a proposição de um checklist baseado nos Fatores Críticos para resultados de projetos de desenvolvimento de software, que, de forma mais precoce, por meio de um tratamento adequado desses Fatores Críticos, viabiliza uma maior probabilidade de sucesso para estes projetos.

\section{Referências}

Ahimbisibwe, A., Caravana, R. Y., \& Daellenbach, U. (2015). A contingency fit model of critical success factors for software development projects. Journal of Enterprise Information Management, 28(1), 7-33.

Chow, T., \& Cao, D. B. (2008). A Survey Study of Critical Factors in Agile Software Projects. Journal of System and Software, 81(6), 961-971.

Hair Jr., J. F., Black, W. C., Babin, B., Anderson, R. E., \& Tatham, R. L. (2009). Análise multivariada de dados (6a ed.). Porto Alegre: Bookman.

Hashim, R., Abbas, M., \& Hashim, M. (2013). Critical success factors assessment in software projects. Science and Information Conference, London, UK.

Kappelman, R., McKeeman, R., \& Zhang, L. (2006). Early Warning Signs of IT Project Failure: The Dominant Dozen. Information Systems Management Journal, 23(4), 31-36.

Kaur, B. P., \& Aggrawal, H. (2013). Critical Failure Factors in Information System: An Exploratory Review. Journal of Global Research in Computer Science, 4(1), 76-82.

Kouzari, E., Gerogiannis, V. C., Stamelos, I., \& Kakarontzas, G. (2015). Critical success factors and barriers for lightweight software process improvement in agile 
development: A literature review. $10^{\text {th }}$ International Joint Conference on Software Technologies, Colmar, Alsace, FR.

Laudon, K., \& Laudon, J. (2010). Sistemas de informações gerenciais (9a ed.). São Paulo: Pearson Prentice Hall.

Lopes, L. F. D. (2016). Métodos Quantitativos. Santa Maria: UFSM.

Marques, A., Varajão, J., Sousa, J., \& Peres, E. (2013). Project Management Success I-C-E model - a work in progress. Procedia Technology, 9, 910-914.

McLeod, L., \& MacDonell, S. G. (2011). Factors that affect software systems development project outcomes: a survey of research. ACM Computing Surveys, 43(4), 24-56.

Müller, F., \& Dal Forno, G. M. B. (2016). Construção e Validação de um Instrumento de Avaliação de Fatores Críticos em Projetos de Software. Anais do Congresso de Administração, Sociedade e Inovação, Juiz de Fora, MG, Brasil. Recuperado em 05 de maio de 2017, de https://even3storage.blob.core.windows.net/anais/37151.pdf.

Nasir, M. H. N., \& Sahibuddin, S. (2011). Critical Success Factors for Software Projects: A Comparative Study. Scientific Research and Essays, 6(10), 21742186.

Pestana, M. H., \& Gageiro, J. (2008). Análise de dados para Ciências Sociais: a complementaridade do SPSS (5a ed.). Lisboa: Edições Silabo.

Pfleeger, S. L. (2004). Engenharia de software: teoria e prática (2a ed.). São Paulo: Prentice Hall.

Rukshan, C. J., \& Mangala, R. P. (2010). Project success factors for Information Technology (IT) related solution deployments; a study conducted for Sri Lankan IT vendors. Fifth International Conference on Information and Automation for Sustainability, Colombo, SRI.

Sandish Group (2013). The chaos manifesto think big, act small. Recuperado em 13 fevereiro, 2015, de www.immagic.com/eLibrary/ARCHIVES/GENERAL/GENREF/S130301C.pdf.

Standish Group (2014). The standish group report chaos. Recuperado em 13 de fevereiro de 2015, de www.projectsmart.co.uk/docs/chaos-report.pdf.

Sudhakar, G. P. (2012). A model of critical success factors for software projects. Journal of Enterprise Information Management, 25(6), 537-558.

Sweis, R. J. (2015). An Investigation of Failure in Information Systems Projects: The Case of Jordan. Journal of Management Research, 7(1), 173-185. 
Verner, J., Sampson, J., \& Cerpa, N. (2008). What Factors Lead to Software Project Failure?. Second International Conference on Research Challenges in Information Science, Marrakech, MAR. 